\title{
Advanced Multiscale Approach (3D-1D) for Hemodynamic Simulation of Blood Flow Properties in Human Arterial Network
}

\author{
Yashar Seyed Vahedein, Yogesh Karnam, Alexander S Liberson \\ Rochester Institute of Technology \\ James E. Gleason Building, 76 Lomb Memorial Drive \\ Rochester, NY 14623-5604, United States \\ Yashar.seyed.vahedein@rit.edu; yk2359@rit.edu; asleme@rit.edu
}

\section{Extended Abstract}

The intricacy of cardiovascular system requires integration of models operating at different levels of complexity. Whereas segments of a high aspect ratio can be delineated using a quasi - one dimensional, non-steady, averaged over the cross section fluid-structure interaction model, the intertwining of such models with more complex three-dimensional ones is imperative when the enhanced local spatial resolution is required. Examples relate to the better simulation, and therefore understanding and diagnosis of the deep venous thrombosis, abdominal aneurism, femoral stenosis, bifurcation areas, etc. The proper detection of a reverse flow (secondary flow) in a local three-dimensional unit helps to predict an arterial decease for large and medium size vessels. Quantification of repetitive mechanical stresses helps to predict the fatigue life of a biological tissue. Analysis of a sensitivity of measured hemodynamic parameters, such as pulse transit time or pulse wave velocity to the geometry and location of a local 3D abnormality allows a proper detection of series of vascular diseases in an affordable way.

In the present work the combination of a commercial 3D and an in-house quasi-1D fluid-structure interaction models is utilized. Mathematical difficulties such as the assignment of boundary conditions at the interface between two models and the development of robust coupling algorithms are resolved using variational approach, specifying a unique set of correct boundary conditions at the 3D/1D interface. Nonlinear geometric model (large deformation) has been utilized to address the enhance compliance of a three-dimensional unit and venous vessels.

\section{Objective}

Successful coupling of 3D-1D models leads to faster numerical models of the arterial network, without facing a significant loss in the accuracy of blood properties prediction comparing to the totally 3D modelling. We aim to:

- Develop a 3D FSI model of a carotid artery bifurcation and perform simulations considering a pulsatile flow, wall anisotropic compliant properties and large deformation in circulatory structure.

- Develop a 1D FSI model of 55 main arteries of the human body to capture the overall flow and BP.

- Derive a coupling methodology at the interface of 3D (local) - 1D (global) models connecting ANSYS workbench to Matlab platform.

\section{Scope}

3D-1D fluid-structure interaction models simulating flow and stress distributions at abdominal aneurism and a bifurcated unit has been presented. Accuracy verification is based on a comparison of a 3D/1D simulation with the totally three dimensional modelling provided by ANSYS - Fluent. Sensitivity analysis of results to the variation of input geometry is presented 